\title{
An empirical framework for tropical cyclone climatology
}

\author{
Nam-Young Kang $\cdot$ James B. Elsner
}

Received: 1 April 2011/Accepted: 24 October 2011/Published online: 13 December 2011

(C) Springer-Verlag 2011

\begin{abstract}
An empirical approach for analyzing tropical cyclone climate is presented. The approach uses lifetimemaximum wind speed and cyclone frequency to induce two orthogonal variables labeled "activity" and "efficiency of intensity". The paired variations of activity and efficiency of intensity along with the opponent variations of frequency and intensity configure a framework for evaluating tropical cyclone climate. Although cyclone activity as defined in this framework is highly correlated with the commonly used exponent indices like accumulated cyclone energy, it does not contain cyclone duration. Empirical quantiles are used to determine threshold intensity levels, and variant year ranges are used to find consistent trends in tropical cyclone climatology. In the western North Pacific, cyclone activity is decreasing despite increases in lifetimemaximum intensity. This is due to overwhelming decreases in cyclone frequency. These changes are also explained by an increasing efficiency of intensity. The North Atlantic shows different behavior. Cyclone activity is increasing due to increasing frequency and, to a lesser extent, increasing intensity. These changes are also explained by a decreasing efficiency of intensity. Tropical cyclone trends over the North Atlantic basin are more consistent over different year ranges than tropical cyclone trends over the western North Pacific.
\end{abstract}

\section{N.-Y. Kang}

Korea Meteorological Administration, Seoul, Korea

N.-Y. Kang $(\varangle) \cdot$ J. B. Elsner

Florida State University, Tallahassee, FL, USA

e-mail: nkang@fsu.edu

J. B. Elsner

e-mail: jelsner@fsu.edu keywords Tropical cyclone climate - Frequency Intensity · Activity · Efficiency of intensity - EOF . Threshold quantile

\section{Introduction}

Tropical cyclones (TCs) inflict serious impacts on national economies (Pielke and Landsea 1998; Zhang et al. 2009). As such, a pressing concern is how climate change might influence them. Numerous studies have been performed largely to try and understand changes in TC activity. In particular there has been an emphasis on defining activity with indices like the Accumulated Cyclone Energy (ACE) and Power Dissipation Index (PDI) (Wu et al. 2006; Camargo et al. 2007; William 2008). Bell et al. (2000) introduced ACE to quantify the activity of TCs per year. ACE is the sum of maximum sustained winds (MSWs) squared every $6 \mathrm{~h}$ during the lifetime of the TC, which gets more sensitive to stronger portion of wind speeds. Likewise, PDI developed by Emanuel (2005), is most sensitive the strongest wind speeds as the values are cubed.

Here we describe PDI (and ACE) as an exponent index (EI) as it depends on TC wind speeds raised to an exponent power. An annual EI is defined as

$\mathrm{EI} \equiv \sum_{\mathrm{N}} \sum_{\mathrm{D}} \mathrm{MSW}^{\mathrm{i}}$,

where $\mathrm{N}$ is storm count, $\mathrm{D}$ is duration using 6-h intervals, MSW is maximum sustained wind speed at each observation, and " $\mathrm{i}$ " is the exponent. Summation convention shows that EI consists of frequency, intensity, and duration. Frequency is the number of TCs per year. Intensity is the MSW per observation and duration is the per cyclone 
lifetime. Thus an annual EI value is an integration over three different physical variables (Emanuel 2007).

Despite some practical uses of an EI, especially for summarizing activity over a season, there remain difficulties in exploiting this framework for understanding the relationships between TCs and climate. Firstly, TC duration is not convenient to deal with. As examined in this paper, duration is positively correlated with intensity since longer lifetimes are more favorable to stronger MSWs. In fact, duration and its mean MSW per cyclone share much of the same information, which can over emphasize variations in these values relative to other variables in the EI. Moreover, duration is not always well defined. Emanuel (2007) remarks that duration is sensitive to observational uncertainties in TC genesis and decay. Secondly, the three variables do not reflect the same concern and the exponent is only applied to the wind speed. Lastly and perhaps most importantly, the variables do not equally contribute to fluctuations in an EI, because they have different averages and different amounts of variability.

In this paper, we suggest an alternative framework for the diagnosis of TC climate. The framework is built on lifetime-maximum wind (LMW) as the metric of TC intensity and it leaves out TC duration. Subsequently, we consider paired time series of annual frequency (FRQ) and annual intensity (INT) that focus on the same wind speed levels. We complete the framework by taking advantage of an empirical orthogonal function (EOF) transformation, where FRQ and INT independently and equally contribute to two new variables labeled "activity" (ACT) and "efficiency of intensity" (EINT).

ACT is the annual value of activity indicating the combined contributions from FRQ and INT, while EINT is the INT portion in ACT which shows how much INT explains ACT. The paired variations of ACT and EINT along with the opponent variations of FRQ and INT configure an empirical framework for examining TC climate. The framework is applied to TCs over the western North Pacific and the North Atlantic. Comparison of results are made between the two regions and with traditional EIs. We find ACT strongly correlated with EI. ACT is decreasing in the western North Pacific, but increasing in the North Atlantic. Despite increases in INT over both basins, the decrease in ACT over the western North Pacific is due to a large decline in FRQ.

The paper is organized as follows. Details of data sets and research domains are described in Sect. 2. FRQ and INT are defined in Sect. 3 along with the concept of threshold quantiles. The EOFs are defined in Sect. 4. The framework is applied to TCs over the western North Pacific and the North Atlantic in Sect. 5. The correlation between variables defined by the framework and EIs are examined in Sect. 6. Time trends in the transformed variables are examined in Sect. 7. A summary of the method and key findings are presented in Sect. 8. All computations and figures were created using R (http://www.r-project.org) and are available upon request from the lead author.

\section{Data and research domains}

\subsection{Best-track data sets}

Table 1 lists the data sources and year ranges for the research conducted here. Separate best-track data sets are obtained from the Joint Typhoon Warning Center (JTWC) and the $\mathrm{NOAA}^{1}$ National Hurricane Center (NHC). Available observations for NHC and JTWC begin in 1851 and 1945, respectively. For this study we use data beginning in 1970, which is generally regarded as the earliest safe year limit to avoid substantial data quality problems, including those associated with the adjustment of satellite observations in the 1960s (Emanuel, 2008). Thus, we extract observations from the two best-track sources over the 40-year period 1970 through 2009, inclusive.

Each track location has a geographic coordinate consisting of a latitude and longitude to the nearest tenth of a degree. Although not particularly relevant for the research conducted here, in cases where the LMW spans more than a single observation, the location of the LMW is taken as the arithmetic average latitude and longitude. Statistics show that the tracks whose LMW span within 24 and $48 \mathrm{~h}$ occupy 86 and $98 \%$ of all tracks in JTWC data and 85 and $96 \%$ of all tracks in NHC data.

\subsection{Geographical domains}

Our interest is in describing and demonstrating a new empirical framework for understanding TC climate. However, the database contains tracks which may not represent the real characteristics of TC climate in the basin interested. Some are cases whose actual genesis locations might be different from observations. Others are the cases whose LMWs might have been influenced by extratropical environment. To limit the potential for contamination of results from unusual TC activity, we restrict our focus to the domains shown in Fig. 1.

The choices of domain bounds are based on the following criteria. The eastern and southern bounds are based largely on the climatological limits of TC genesis. In the North Pacific the eastern bound is also determined so as to exclude TCs whose genesis locations are near the international date line $\left(180^{\circ} \mathrm{E}\right)$ as they are likely to have

\footnotetext{
$\overline{1}$ National Oceanic and Atmospheric Administration.
} 
Table 1 Best-track data sources and year ranges

\begin{tabular}{lll}
\hline Source & Ocean basin & Year range (length) \\
\hline JTWC & Western North Pacific & 1970-2009 (40 years) \\
NHC & North Atlantic & $1970-2009$ (40 years)
\end{tabular}

JTWC is the Joint Typhoon Warning Center and NHC is the NOAA National Hurricane Center

originated in the central or eastern North Pacific. The western bound in both basins is limited by land masses.

The northern boundary is based on sea-surface temperature (SST). A SST value of $26.5^{\circ} \mathrm{C}$ is generally considered the minimum value for the growth of tropical convection (Gray 1968; Graham and Barnett 1987), so the northern bound is set as the average northward extension of this threshold SST value. While some TCs can reach their LMW north of this boundary, especially over the North Atlantic, intensification mechanisms in these relatively rare cases are often associated with mid-latitude influences that are not of interest here. Based on the above considerations, we fix the domain bounds of the western North Pacific region to be $104-175^{\circ} \mathrm{E}, 3-35^{\circ} \mathrm{N}$, and that of the North Atlantic to be $260-342^{\circ} \mathrm{E}, 9-40^{\circ} \mathrm{N}$.

\subsection{TC selection}

Each TC is defined by attributes including basin name, genesis date, genesis location, lifetime-maximum intensity location, lifetime-ending location, LMW and duration. Here we consider only TCs that reached the threshold intensity of $17 \mathrm{~m} \mathrm{~s}^{-1}$. TCs that failed to reach this intensity are removed from further consideration. We then consider the TCs whose genesis and lifetime-maximum intensity locations are within the domain (Fig. 1). In this paper, those included TCs are designated as TS2TY. Excluded TCs outside the domain though their lifetime-maximum intensity exceed $17 \mathrm{~m} \mathrm{~s}^{-1}$ are also shown in Fig. 1. The remainder of the paper considers only TS2TY.

\section{Frequency and intensity}

\subsection{Intensity}

Figure 2 shows scatter plots of average lifetime MSW versus TC duration for all TS2TY over the western North Pacific and North Atlantic domains from data spanning the 40-year period (1970-2009). The plots indicate a strong correlation between TC duration and intensity. The Pearson correlation coefficients are $0.72 \pm 0.02$ (s.e.) and $0.64 \pm 0.04$ (s.e.) using the JTWC and NHC data, respectively. The significant correlation supports our decision to remove duration as it represents redundant information once a measure of TC intensity is included.

In addition, the correlation between maximum MSW per cyclone, that is LMW, and average MSW per cyclone is
Fig. 1 Research domains. The domains are outlined with white lines. Tracks of the included TCs are shown in panel (a) and tracks of the the excluded TCs are shown in panel (b). Genesis location is shown with a green circle and the location of lifetime maximum intensity (LMW) with a red circle. Tracks are represented as straight lines between genesis and LMW locations and between LMW locations and lysis locations
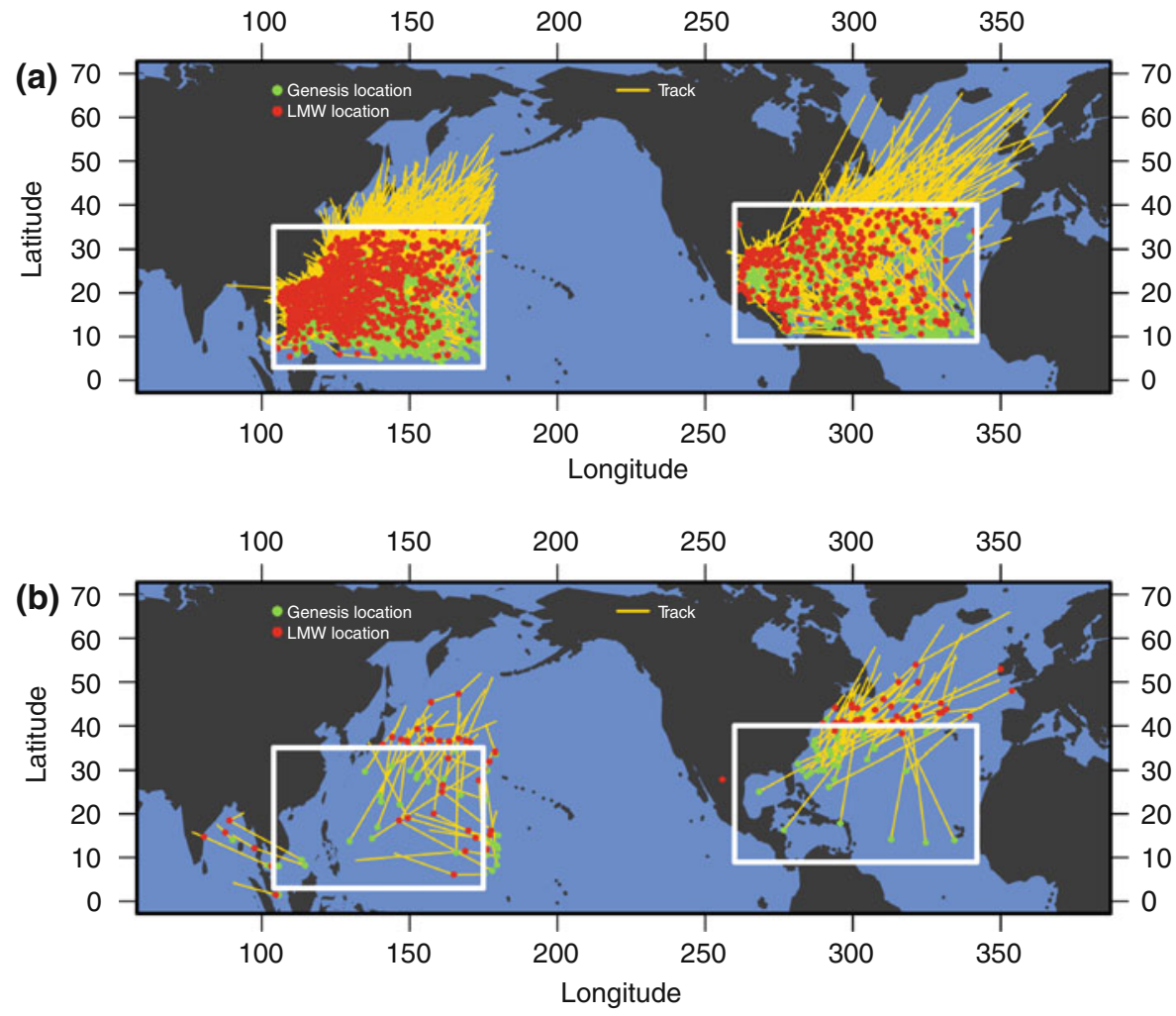
Fig. 2 Scatter plots of mean MSWs versus durations per cyclone in a the western North Pacific and $\mathbf{b}$ the North Atlantic over 40 years (1970-2009). JTWC best-tracks and NHC best-tracks are used in the corresponding basin. The ordinary least-squares regression line is shown in green

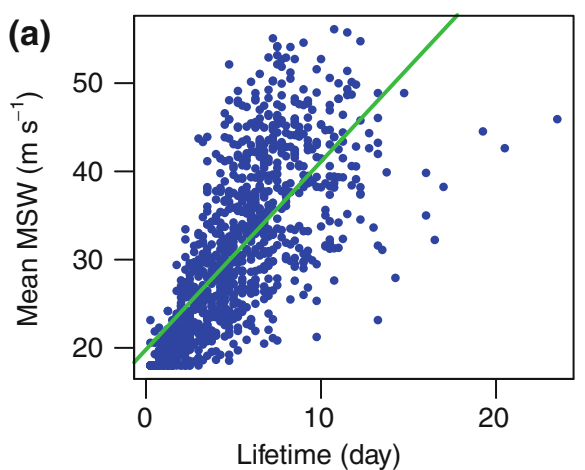

examined from the same track data used in Fig. 2. The result shows $0.96 \pm 0.01$ (s.e.) and $0.94 \pm 0.02$ (s.e.), respectively. Accordingly, LMW is a reasonably good metric for TC intensity. Given LMW as a metric of TC intensity, we compute the annual average TC intensity as

Annual intensity $=\int_{\text {LMW }} \mathrm{x} \cdot \mathrm{f}(\mathrm{x}) \mathrm{dx}$,

where $f(x)$ is the probability density function of LMW. The annual intensity is interpreted as the annual average LMW. Annual intensity can be considered independent of annual frequency since annual intensity can be obtained when threshold exceedance levels of LMWs are summed and divided by annual frequency.

\subsection{Orthogonal variations of frequency and intensity}

To simplify notation, annual frequency and annual intensity are denoted as FRQ and INT, respectively. Figure 3 explains the orthogonal relationship between FRQ variation and INT variation using the gamma distribution. The gamma distribution express continuous probability densities, which are parameterized by a shape and a scale parameter. Here, the plot is made by fitting a gamma distribution to the LMW for the TS2TY in the JTWC data set. All those curves are for the shape parameter of 1.44. Then the distribution is multiplied by a random variable describing the annual frequency of TCs (FRQ) as shown in thin lines. Thin lines are the gamma distribution with increasing values of FRQ. Thick lines are the same distribution of LMW when the FRQ is 25. The blue curves are derived from the black curves by multiplying the scale parameter by 1.83 .

The set of blue curves represents a different distribution of INT relative to the black curves indicating a greater ratio of strong to weaker TCs. FRQ variation is illustrated by changes in the height of the curves while INT variation is illustrated by changes in the width of the curves (compare the black curves with the blue curves). Given the same FRQ, larger width represents larger INT indicating larger

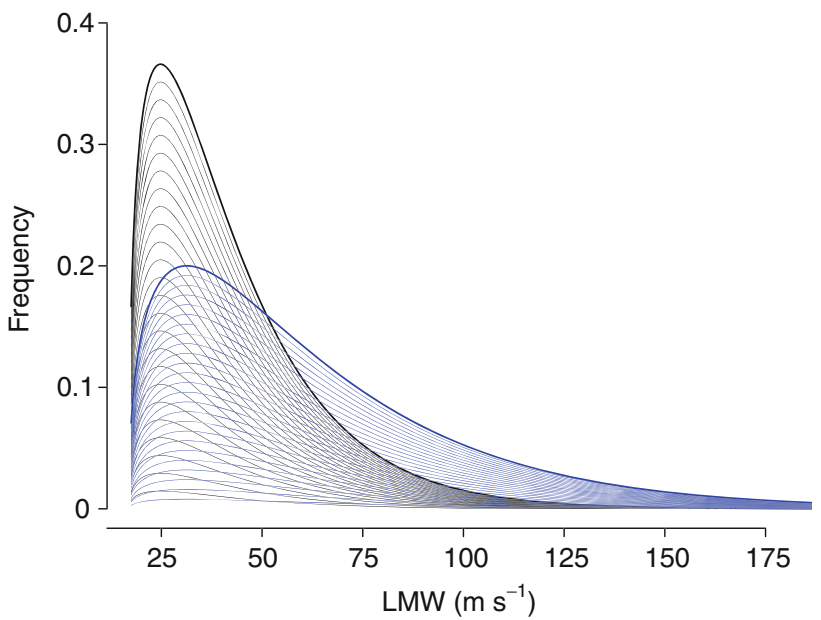

Fig. 3 Hypothetical distribution of LMW. Curves are constructed by multiplying the gamma distribution by a count. The set of black curves is based on data from the JTWC (1970-2009) and a gamma fit with shape and scale parameters 1.44 and 34.6 , respectively. The blue curves are derived from the black curves by multiplying the scale parameter of the gamma distribution by 1.83

average of LMWs. Thus we can consider variations of FRQ and INT as independent following orthogonal directions.

\subsection{Threshold quantiles}

Having established a coordinate system for the TC data, we prepare annual time series of FRQ and INT. FRQ-INT pairs are available at various threshold values of LMW. That is, for a given value of LMW we can count the number of TCs that exceed this threshold.

Here we suggest using quantiles as a way of analysis. Previous studies (Jagger and Elsner 2008; Elsner et al. 2008) have shown the utility of the quantile approach in examining TC intensity. As used here, a quantile is the value of LMW for which the quantile percentage of all TCs have an LMW less than this value. For example if the 0.8 quantile (80th percentile) LMW is $55 \mathrm{~m} \mathrm{~s}^{-1}$, this means that $20 \%$ of all TCs have LMW larger than $55 \mathrm{~m} \mathrm{~s}^{-1}$. The $20 \%$ can be converted to a count, so that for each LMW 
quantile there is a frequency of TCs above this value. Here we consider FRQ as the total number of TCs above this quantile value. We call this quantile value the threshold quantile $(\tau)$, and we define the INT as a function of $\tau$ by

$\operatorname{INT}(\tau)=\int_{\operatorname{LMW}(>\tau)} x \cdot f(x) d x$,

where again $f(x)$ is the probability density function of LMW.

Having defined our FRQ and INT variables, next we consider how to use them to construct an orthogonal framework for describing annual variability in TC climate.

\section{A framework for TC climate analysis}

The framework is based on an empirical orthogonal function (EOF) transformation of the FRQ and INT variables defined in the previous section. In short, from the two variables INT and FRQ, we construct two new orthogonal variables called ACT and EINT. Figure 4 shows a schematic of the framework. Frequency is on the vertical axis and intensity is on the horizontal axis. Points in the upper-right quadrant are characterized by high values of frequency and intensity and points in the lowerleft quadrant are characterized by low values of frequency and intensity. Thus a positive diagonal provides an axis that captures the variability in TC activity that we denote ACT. Orthogonally, points in the upper-left quadrant are characterized by high values of frequency and low values of intensity and points in the lower-right quadrant are characterized by low values of frequency and high values of intensity. Thus a negative diagonal provides an axis that captures the variability in the efficiency of intensity that we denote EINT.

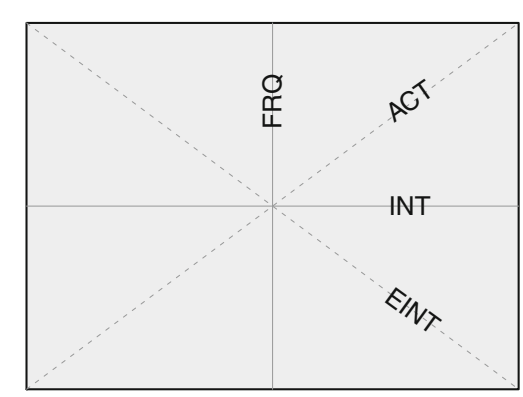

Fig. 4 Schematic of our TC climate framework. Paired values of FRQ and INT are plotted in the two-dimension cartesian space. The positive diagonal (positive slope) captures the level of coherency between FRQ and INT and is labeled ACT. The negative diagonal (negative slope) captures the level of discordancy between FRQ and INT and is labeled EINT
ACT is computed as

$\mathrm{ACT}=\left(\frac{\mathrm{INT}-\mu_{\mathrm{INT}}}{\sigma_{\mathrm{INT}}}+\frac{\mathrm{FRQ}-\mu_{\mathrm{FRQ}}}{\sigma_{\mathrm{FRQ}}}\right) / \sqrt{2}$,

where INT and FRQ are vectors of annual values, $\mu$ and $\sigma$ denote their respective mean and standard deviations. Therefore ACT is a linear combination of INT and FRQ using standardized values. By this construction ACT is an eigenvector. The eigenvector indicates the magnitude and direction of the coincidence in FRQ and INT. Remembering that an EI is the product of variables having different magnitudes and scales, ACT variation is not the same as variation of TC kinetic energy, which an EI captures.

Similarly, EINT is computed as

$$
\mathrm{EINT}=\left(\frac{\mathrm{INT}-\mu_{\mathrm{INT}}}{\sigma_{\mathrm{INT}}}-\frac{\mathrm{FRQ}-\mu_{\mathrm{FRQ}}}{\sigma_{\mathrm{FRQ}}}\right) / \sqrt{2},
$$

where, as before, INT and FRQ are vectors of annual values, $\mu$ and $\sigma$ denote their respective mean and standard deviations. Like ACT, EINT is a linear combination of INT and FRQ and by construction is a vector indicating the degree to which variations in FRQ and INT are out of phase. Mathematically, EINT explains the portion of INT in ACT. Although we denote this eigenvector as the efficiency of intensity, its additive inverse could be denoted as the efficiency of frequency.

Consider a single-handed lever on a kitchen faucet as an analogy. Moving the lever to adjust water flow is analogous to variations in ACT, whereas moving the same lever to adjust water temperature is analogous to variations in EINT. Rotated $45^{\circ}$ from the FRQ and INT axes, the ACT and EINT axes provide an untapped coordinate reference for capturing variability in TC climate.

\section{TC activity and efficiency of intensity}

Here we apply the framework defined in the previous section using TC data from the western North Pacific and the North Atlantic basins over the 40-year period (1970-2009). The data from the western North Pacific are from the JTWC and the data from the North Atlantic are from the NHC. Annual values of FRQ and INT, that is annual TC frequency and annual average TC intensity, are computed separately for each basin. These are based on LMW observations which means lifetime-maximum wind speeds.

Annual values of ACT and EINT are computed at each $\tau$ (threshold quantile). For instance, at $\tau=0.5$, we have a median LMW and we compute INT( 0.5$)$ as the summation of LMW over all LMW exceeding this median value, and FRQ $(0.5)$ as the number of summations. From the set of paired values of $\operatorname{FRQ}(0.5)$ and $\operatorname{INT}(0.5)$ we compute 
$\operatorname{ACT}(0.5)$ and $\operatorname{EINT}(0.5)$ from Eqs. 4 and 5. The computations are performed separately for each year to generate 40 values of $\operatorname{ACT}(0.5)$ and 40 values of $\operatorname{EINT}(0.5)$. We then rank each year according to the year's component value and convert the values to a probability using the empirical cumulative density function. Thus, a given $\tau$ year's rank according to its ACT value is independent of its $\tau$ year's rank according to its EINT value.

Results of this procedure are shown in Fig. 5. Panels (a) and (b) are for the western North Pacific and display contours of ranked probabilities for ACT and EINT, respectively. Threshold quantile values and corresponding average LMW $\left(\mathrm{m} \mathrm{s}^{-1}\right)$ are plotted on the vertical axis. Contours below the median are shaded in blues and contours above the median are shaded in yellows through red. Contour lines are nearly vertical as a consequence of the integration used in defining FRQ and INT.

The individual contour values are interpreted as follows. Consider panel (a) showing ACT. At the median threshold quantile ( 0.5 value along the vertical axis) the ranked probability for 1990 is about 0.8 (light red). This indicates that the median activity level (as defined by FRQ and INT) during 1990 was well above the 40-year median level. In contrast, consider panel (b) showing EINT. At the same median threshold quantile, the ranked probability for the same year is 0.3 indicating that 1990 was below the 40-year median in terms of efficiency of intensity.

The pattern shows relatively high ranking of ACT in the early 1970s followed by an extended period of low values until the late 1980s. The early 1990s featured high activity then low activity again in the late 1990s closer to 2000. While it was relatively active in the early 2000s, there has been a recent decline in ACT. The pattern of EINT is somewhat different featuring a broad tendency for increasing efficiency of intensity over the decades. The efficiency of intensity introduces a concept of variability between a few strong TCs and many weak TCs. The result shows diagnostics of those variations. Recent EINT peaks indicate the variability which returns stronger but fewer TCs are favorable given the same TC energy. Thus we find that the recent fewer TCs are explained by increases in the efficiency of TC intensity. Fewer TCs don't necessarily imply a decrease in ACT, but the recent lull in ACT are found to have been caused mainly by fewer TCs. We also note tendency for a quasi-periodic fluctuation on the sub-decadal timescale in values of EINT, but not in ACT.

The same methodology reveals a different TC climate for the North Atlantic (Fig. 6). Here we see a general increasing trend in ACT attributable to coherent upward trends in FRQ and INT. However, there is a corresponding decreasing trend in EINT attributable to the fact that FRQ increases are greater than INT increases. Also in contrast to the TC climate of the western North Pacific, on top of the general trends there appears to be a quasi-periodic fluctuation in ACT. Though some fluctuations are also found in EINT, the amplitudes are not quite clear to discern periodicity. Furthermore the threshold quantile levels correspond to weaker LMW over the North Atlantic compared to the western North Pacific. On average, the difference is $11 \mathrm{~m} \mathrm{~s}^{-1}$ for the upper $25 \%(\tau=0.75)$ of the LMWs confirming the well-known fact that TCs over the western North Pacific are stronger, on average, than TCs over the North Atlantic.
Fig. 5 Ranked probabilities of annual ACT (a) and EINT (b) for TCs over the western North Pacific (see Fig. 1). The rankings are done separately for ACT and EINT for each threshold quantile $(\tau)$. Average LMW over all years for each $\tau$ are shown on the right axes. Contours are colored blue for rankings below the mean and yellow to red for rankings above the median at intervals of 0.1 .

Data are from the JTWC for the period 1970-2009
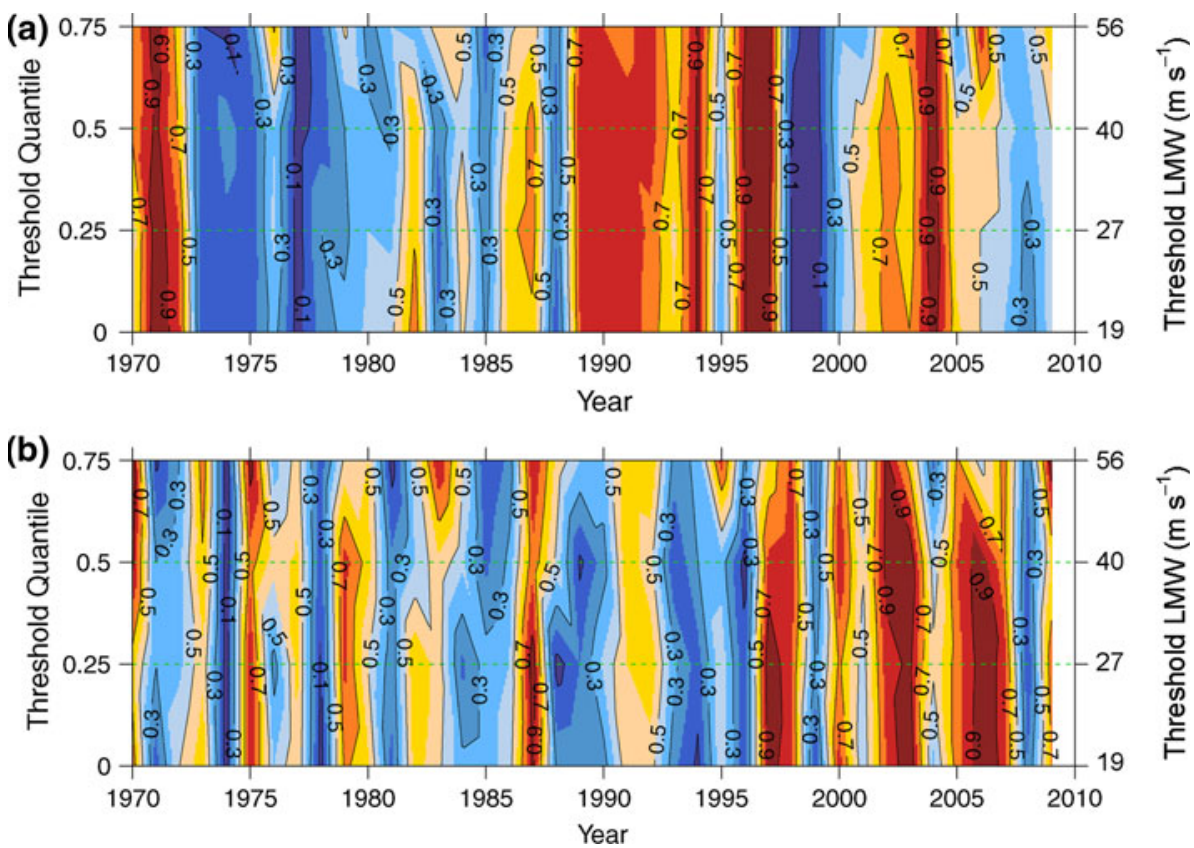
Fig. 6 Ranked probabilities of annual ACT (a) and EINT (b) for TCs over the North Atlantic (see Fig. 1). Data are from the NHC for the period 1970-2009. Colors and labels are the same as in Fig. 5
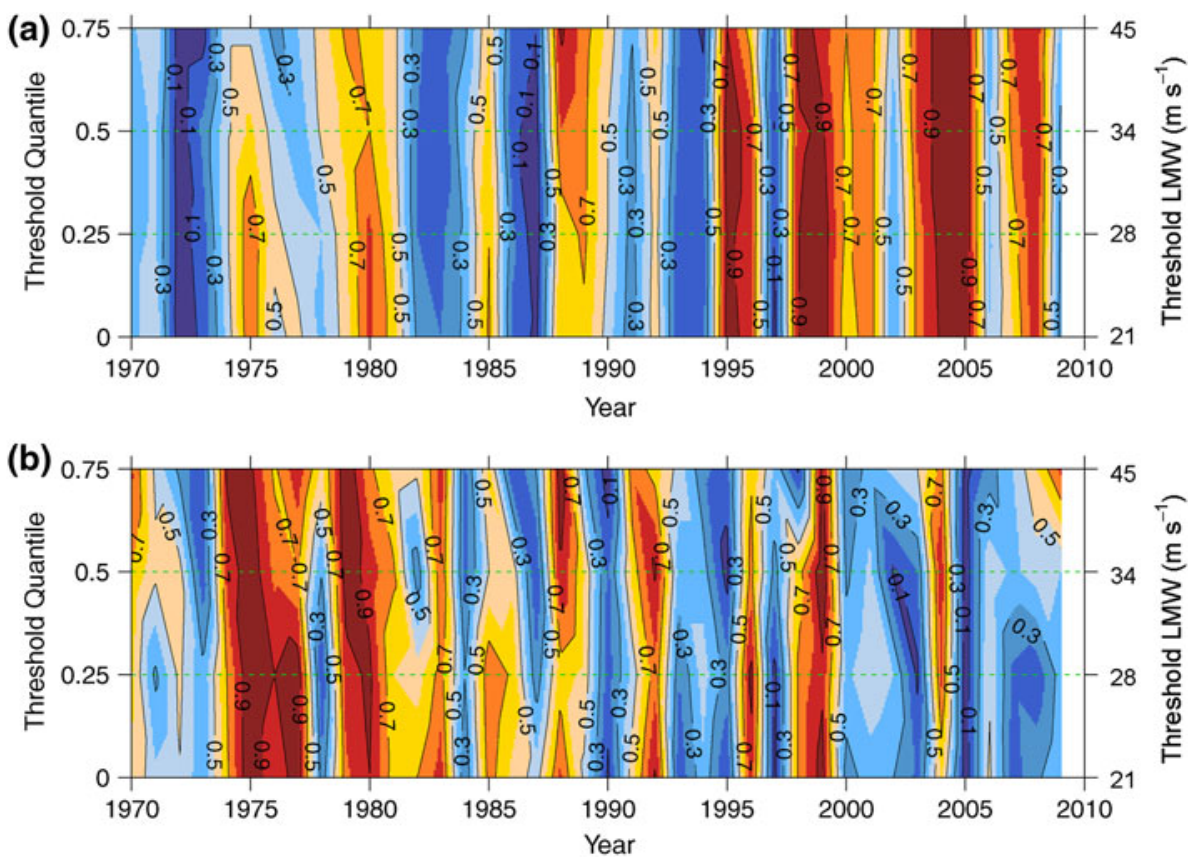

Differences in TC climatology between the two basins can also be seen by examining the percentage of combined variance of FRQ and INT explained by ACT and EINT (Table 2). With two variables the total variance explained by the EOFs is $100 \%$. We can see that over the western North Pacific at most of quantile thresholds the variance explained by EINT is near or slightly greater than the variance explained by ACT. Mean variance explained by EINT shows that EINT is 1st mode of variance in FRQ and INT. This contrasts rather markedly with conditions over the North Atlantic. Here at all quantiles, and by a larger margin, ACT explains more of the variance in FRQ and INT than does EINT. In this basin, ACT takes the role of 1st mode. As shown in the two basins, other basins may also have distinct characteristics.

Table 2 EOF variance

\begin{tabular}{lccccl}
\hline Statistic & \multicolumn{5}{l}{ Threshold quantile } \\
\cline { 2 - 6 } & 0 & 0.25 & 0.5 & 0.75 & Mean \\
\hline \multicolumn{4}{l}{ Western } & North Pacific \\
ACT & 51.1 & 41.0 & 45.3 & 43.2 & 45.1 (2nd mode) \\
EINT & 48.9 & 59.0 & 54.7 & 56.8 & 54.9 (1st mode) \\
North Atlantic & & & & \\
ACT & 62.4 & 57.8 & 62.3 & 68.2 & 62.7 (1st mode) \\
EINT & 37.6 & 42.2 & 37.7 & 31.8 & 37.3 (2nd mode) \\
\hline
\end{tabular}

Percent of the combined variance of FRQ and INT explained by ACT and EINT
These differences suggest the need for different interpretations of seasonal forecasts. Forecasts calling for a large number of TCs in the North Atlantic should infer a prediction of high TC intensities. In contradistinction, a similar forecast for high TC activity in the western North Pacific might not imply a prediction of high TC intensities.

\section{Correlation between ACT and EI}

As defined above, ACT is the normalized sum of FRQ and INT and, as shown, can be defined at various threshold quantiles of LMW. As such, it is interesting to examine the correlation between annual values of ACT and annual values of an EI (exponent index).

Correlations between ACT and the two commonly-used EIs are shown in Table 3 using four threshold quantiles. Exponent index 2 (EI2) and exponent index 3 (EI3), where the number indicates the exponent, are ACE and PDI, respectively. The indices are computed annually using all MSWs along the path of TS2TY. EI2 and EI3 values are converted into ranked probabilities using the empirical cumulative distribution function as was done with ACT.

The large correlations indicate a tight relationship between ACT and ACE and PDI computed from TCs over the western North Pacific and the North Atlantic. Over the North Atlantic, largest correlations are noted when the strongest $50 \%$ of all TC observations are included. In contrast, over the western North Pacific correlations are larger when weaker observations are included ( $\tau=0$ and 0.25 ). 
Table 3 Correlations between ACT and EIs. EI2 is the ACE and EI3 is the PDI

\begin{tabular}{lcccc}
\hline \multirow{2}{*}{ ACT } & \multicolumn{4}{l}{ Threshold quantile } \\
\cline { 2 - 5 } & 0 & 0.25 & 0.5 & 0.75 \\
\hline Western North & Pacific & & & \\
EI2 & $\mathbf{0 . 9 5}$ & $\mathbf{0 . 9 5}$ & 0.92 & 0.82 \\
EI3 & $\mathbf{0 . 9 2}$ & $\mathbf{0 . 9 2}$ & 0.91 & 0.84 \\
North Atlantic & & & & \\
EI2 & 0.89 & 0.90 & $\mathbf{0 . 9 3}$ & 0.89 \\
EI3 & 0.91 & 0.92 & $\mathbf{0 . 9 5}$ & 0.92 \\
\hline
\end{tabular}

The largest correlation with the EI is shown in bold

Moreover, it is natural that the correlations between ACT and EI3 in the North Atlantic appear larger than in the western North Pacific. This is because ACT will have larger correlation with EI3 than EI2 when more INT contributes to ACT. From Table 3 we may infer the larger correlations between INT and ACT in the North Atlantic than in the western North Pacific.

As with seasonal forecasts, these results suggest the interpretation of ACE and PDI as indicators of TC climate are different depending on where the TCs occur.

\section{Trends}

During the past decades, many researchers produced numerical simulations of $\mathrm{TC}$ with the expectation of
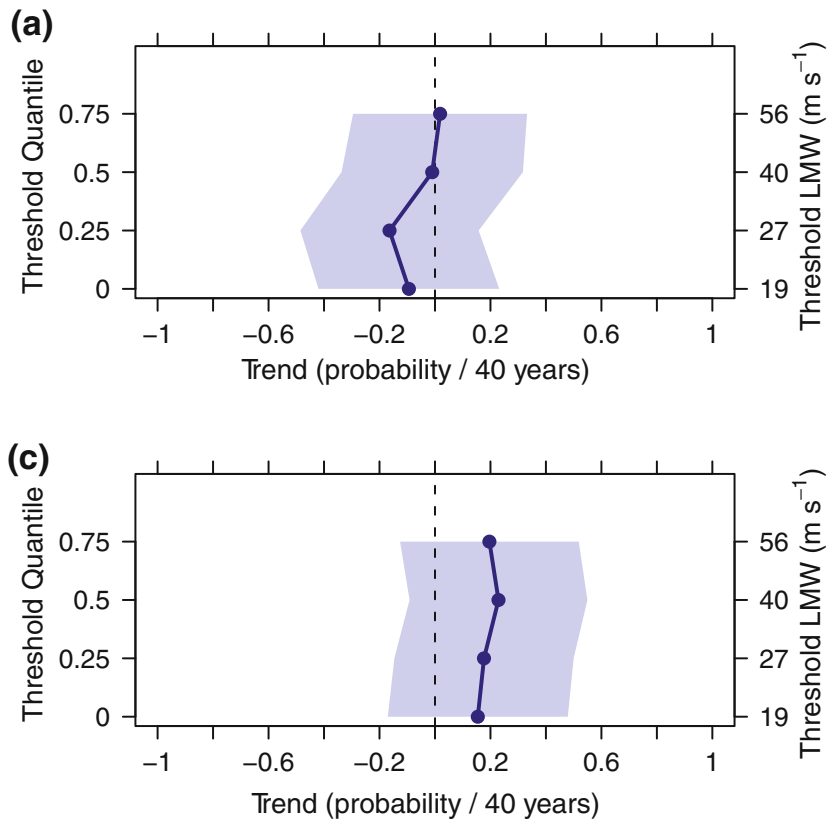

Fig. 7 Trends in TC climate over the western North Pacific. Trends in units of ranked probability per 40 years are shown for a FRQ, b INT, c ACT, and d EINT. The quantile threshold is labeled on the understanding how global warming and SST increases will lead to changes in number and intensity (Broccoli and Manabe 1990; Haarsma et al. 1993; Krishnamurti et al. 1998). However, numerical approach still may not present reliable consensus of trends, depending on the model characteristics (Chan and Liu 2004).

Here we quantify the long term trends in FRQ, INT, ACT and EINT in both TC basins. Trends are examined using data over the period 1970-2009. Trend estimates of TC variables in the western North Pacific are made using data from the JTWC. Trends are computed using ordinary least-squares regression at each threshold quantile.

Results for the western North Pacific are shown in Fig. 7. Trends have units of ranked probability per 40 years. A positive (upward) trend in ranked probability indicates a tendency for the later years to be among the most extreme relative to the earlier years. The shaded region delineates the point-wise $95 \%$ confidence intervals about the mean trend estimates.

Results show no trends in FRQ, but small upward trends in INT, ACT, and EINT. Statistically significant upward trends are noted at the three highest threshold quantiles for INT, but not for ACT and EINT.

In the North Atlantic things are different (Fig. 8). Highly significant upward trends in FRQ are apparent at all threshold quantiles. Significant upward trends in the higher threshold quantiles of INT are also noted. These upward trends in FRQ and INT translate to significant upward trends in ACT. In contrast, we note significant downward
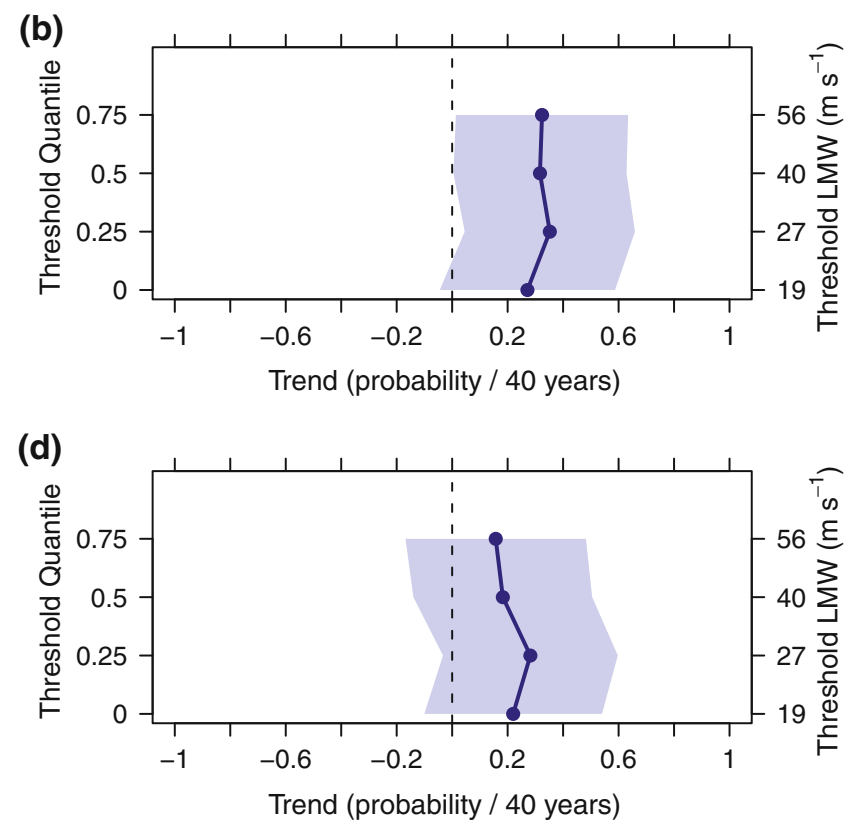

left vertical axis and the corresponding threshold LMW $\left(\mathrm{m} \mathrm{s}^{-1}\right)$ is labeled on the right vertical axes. Data are from the JTWC for the period 1970-2009 

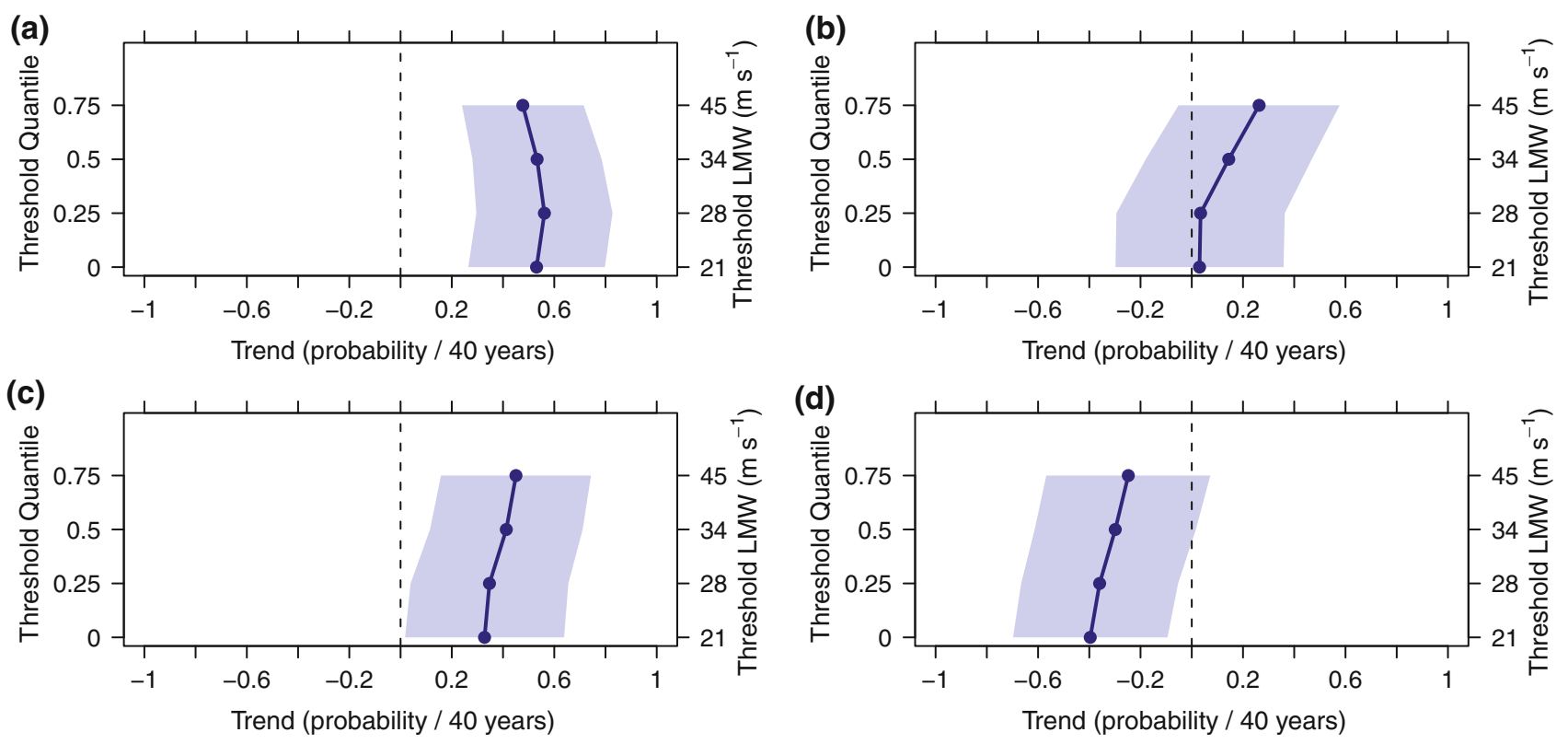

Fig. 8 Trends in TC climate over the North Atlantic. Data are from the NHC for the period 1970-2009. The blue lines are trends. Labels are the same as in Fig. 7

trends in EINT as the strong upward trends in FRQ dominate the more modest upward trends in INT.

Overall we find upward trends in TC activity in both basins. However, in the western North Pacific the trends are isolated to increases in the efficiency of intensity, whereas in the North Atlantic the upward trends in activity are the most dominant.

Since trends depend to some extent on the range of years over which they are estimated, it is interesting to examine the estimates using different start years. Figure 9 shows trends in FRQ, INT, ACT, and EINT computed over different year ranges by varying the start year. The trends are for the 0.5 threshold quantile values using western North Pacific TCs. Here the unit of change is rank probability per 10 years. Trends are shown for the JTWC data. The 95\% confidence intervals are shaded. Confidence limits enlarge as the number of years goes down largely because of decreasing sample size.

FRQ shows clear downward trends when estimated over the last 30 years or so. In contrast, upward trends are noted in INT that are insensitive to the start year. The downward trends in FRQ, the magnitude of which are sensitive to start year, are reflected in ACT. Large upward trends in EINT are noted, with the magnitude of the trend increasing over the last few decades.

TC climate trends in the North Atlantic over different year ranges at 0.5 threshold quantile are shown in Fig. 10. The North Atlantic storm climate gives relatively clear and constant trends over different year ranges. Significant increasing trends over 0.1 per 10 years are seen in FRQ.
The most recent set of 20 years (1990-2009) shows more apparent values up to 0.2 per decade. FRQ trends are consistent with the diagnostic results of Webster et al. (2005) showing the North Atlantic is the only basin where increasing cyclone occurrence is taking place. Together with increasing trends of INT, FRQ increases contribute to upward trends in ACT. EINT explains that FRQ contributions are more dominant than INT contributions over all year ranges. Overall, in the North Atlantic, activity is going up as frequency and intensity increase, with frequency increases relatively larger.

\section{Summary}

An empirical TC climate framework consisting of four variables was constructed. Construction starts by defining threshold quantile frequencies and intensities using lifetime maximum wind and continues by applying an EOF analysis to the variables yielding new quantities related to activity and efficiency of intensity. A synopsis of the main work done on the framework and its difference with the classical framework of PDI and ACE is:

- TC duration is removed for its uncertainty and redundancy. We confirm that per-cyclone duration contains largely the same information as per-cyclone intensity.

- TC intensity is replaced by LMW, which can be considered as having the least accumulation of observational errors. 
Fig. 9 Trends in TC climate over the western North Pacific as a function of start year. Trends in units of ranked probability per 10 years are shown for a $\operatorname{FRQ}(0.5), \mathbf{b}$ $\operatorname{INT}(0.5)$, c ACT(0.5) and d EINT(0.5). The blue lines are trends based on the collection of TCs in the JTWC data set. The start year for the trend estimate is shown along the horizontal axis on the bottom and the number of years in the data set is shown along the horizontal axis at the top

Fig. 10 Trends in TC climate over the North Atlantic as a function of start year. Data are from the NHC. The blue lines are trends. Labels are the same as in Fig. 9
Data range (year)

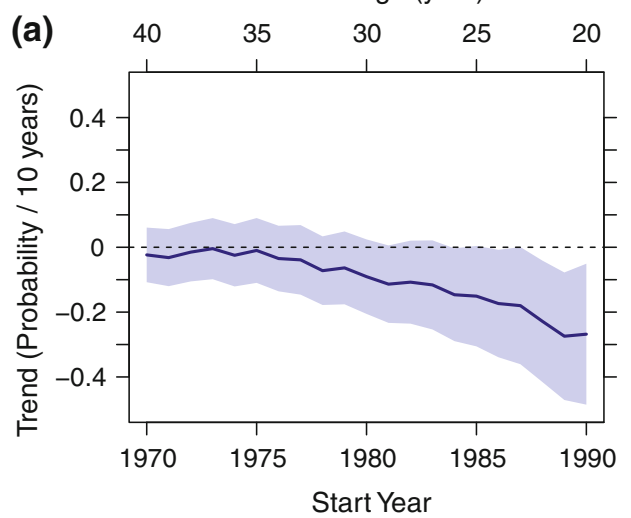

Data range (year)

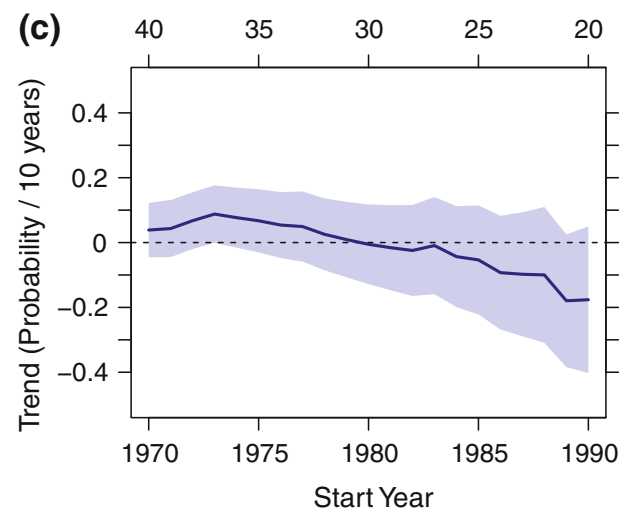

Data range (year)

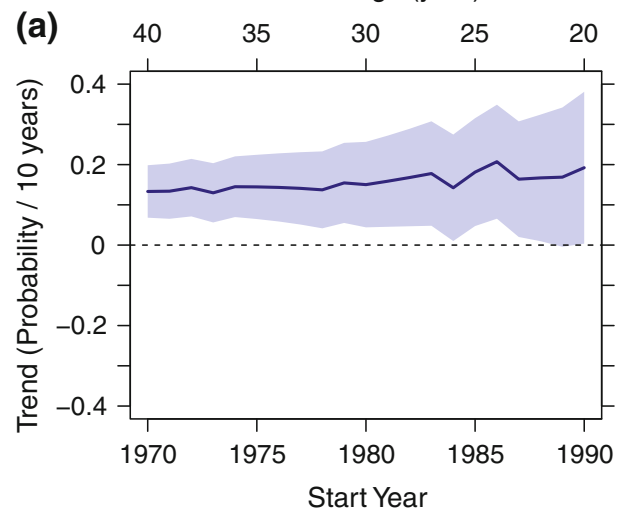

Data range (year)

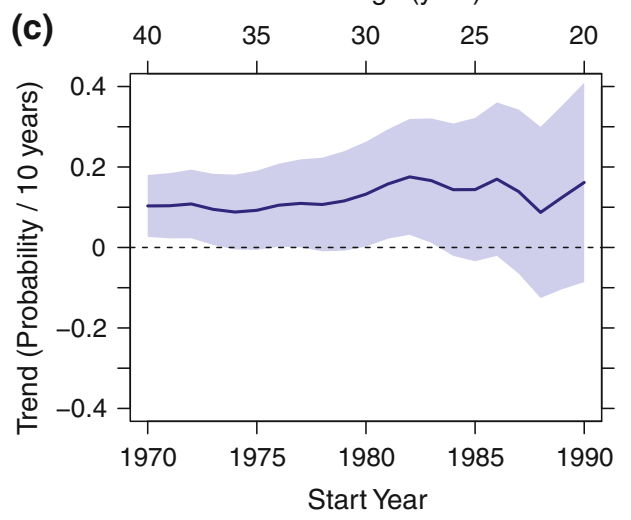

Data range (year)

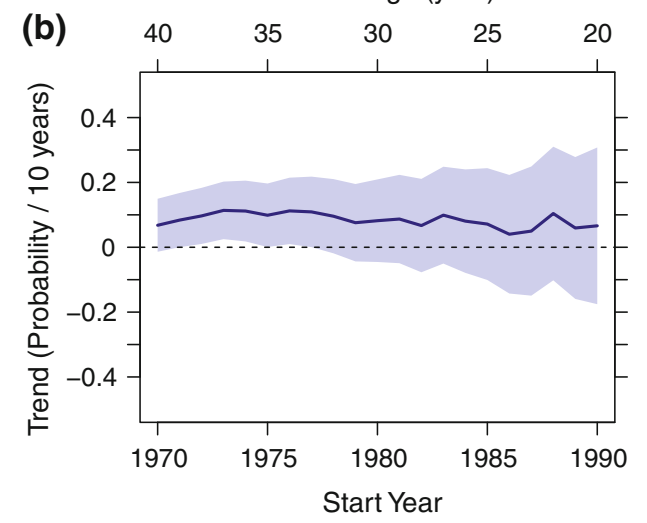

Data range (year)

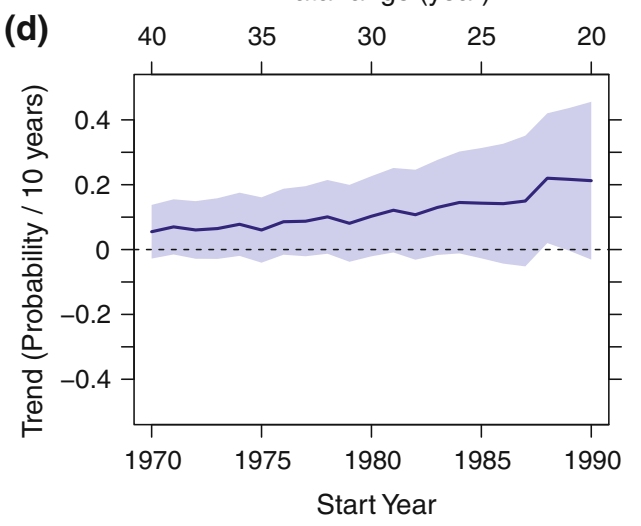

Data range (year)

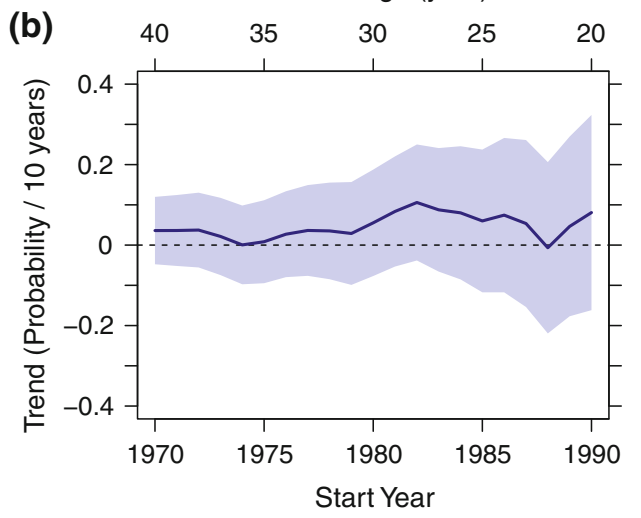

Data range (year)

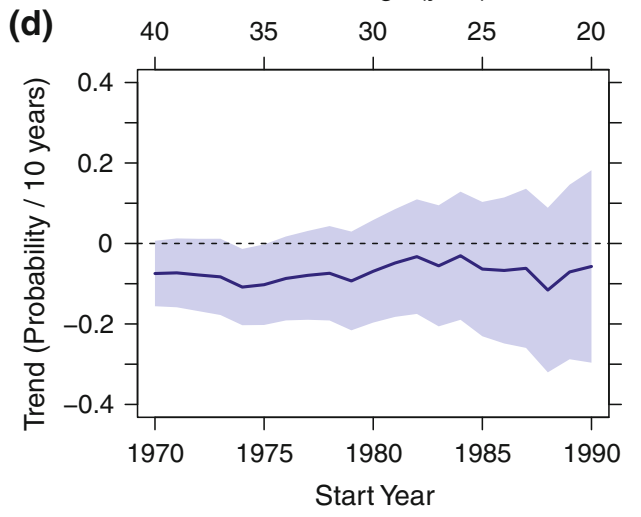


- Research domains are used to enhance the diagnostics of TCs in the western North Pacific and the North Atlantic. TCs are selected for the cases whose LMWs exceed $17 \mathrm{~m} \mathrm{~s}^{-1}$ and the genesis locations and maximum-intensity locations occur within the domain.

- FRQ and INT are used to induce ACT and EINT through an EOF analysis. The four variables exist in a two-dimension space so a given TC climate state can be represented as a single location relative to the four variables.

- We extend the analysis to various intensity levels using threshold quantiles of LMW. This method reveals some ambiguity when using the classical framework of PDI and ACE to summarize seasonal activity.

- Trends in the TC variables are examined at different quantile thresholds and for different TC data bases.

Using this new framework, TC climate over the western North Pacific and the North Atlantic was diagnosed. A summary of the results are:

- Over the past 40 years, variations in TC climate in the western North Pacific appear to be quite a bit different that variations in TC climate in the North Atlantic. The differences cannot be easily captured using ACE or PDI.

- In the western North Pacific storm activity is going downward. Though intensity is increasing, declining frequency is more than enough to offset it. These variations are also explained by the increasing efficiency of intensity. A schematic summary of these trends is given in Fig. 11.

- In the North Atlantic storm activity is going upward. This is made clear by increasing frequency and increasing intensity, with the increasing frequency out

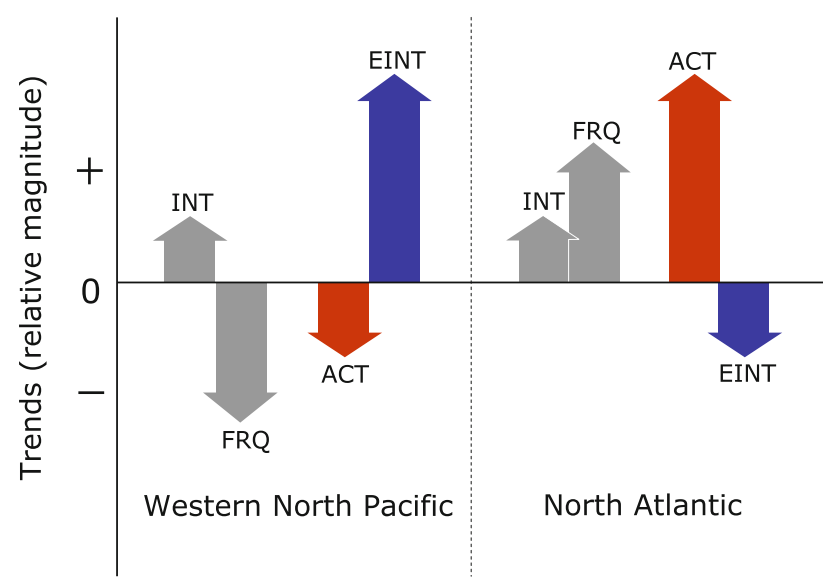

Fig. 11 Schematic of trends in TC climate over the western North Pacific and the North Atlantic. Relative magnitude indicates increasing $(+)$ or decreasing $(-)$ trends over the years pacing the increasing intensity. These variations are also explained by the decreasing efficiency of intensity (see Fig. 11).

Though we built the framework by first defining FRQ and INT and then extracting ACT and EINT, how nature works might be reversed. That is, nature might regulate ACT and EINT, which gets recorded as changes in FRQ and INT. The search is now on to find environmental factors that best correspond to these TC climate variables as a first step toward unraveling the causal mechanisms in TC climatology. At a minimum we expect research using this new framework to help improve our understanding of TC climate.

Acknowledgments The work was supported by the Korean Meteorological Administration through a visiting fellowship to the lead author.

\section{References}

Bell GD et al (2000) Climate assessment for 1999. Bull Am Meteorolog Soc 81:1328

Broccoli AJ, Manabe S (1990) Can existing climate models be used to study anthropogenic changes in tropical cyclone climate? Geophys Res Lett 17:1917-1920

Camargo SJ, Emanuel K, Sobel AH (2007) Use of a genesis potential index to diagnose ENSO effects on tropical cyclone genesis. J Clim 20:4819-4834

Chan JCL, Liu KS (2004) Global warming and western North Pacific typhoon activity from an observational perspective. J Clim 17:4590-4602. doi:10.1175/3240.1

Elsner JB, Kossin JP, Jagger TH (2008) The increasing intensity of the strongest tropical cyclones. Nature 455:92-95. doi:10.1038/ nature 07234

Emanuel KA (2005) Increasing destructiveness of tropical cyclones over the past 30 years. Nature 436:686-688

Emanuel KA (2007) Environmental factors affecting tropical cyclone power dissipation. J Clim 20:5497-5509

Emanuel KA (2008) The hurricane-climate connection. Bull Am Meteorolog Soc 89:ES10-ES20. doi:10.1175/BAMS-895-Emanuel

Graham NE, Barnett TP (1987) Sea surface temperature, surface wind divergence, and convection over tropical oceans. Science 238:657-659

Gray WM (1968) Global view of the origin of tropical disturbances and storms. Mon Wea Rev 96:669-700

Haarsma RJ, Mitchell JFB, Senior CA (1993) Tropical disturbances in a GCM. Climate Dyn 8:247-257

Jagger TH, Elsner JB (2008) Modeling tropical cyclone intensity with quantile regression. Int J climatol. doi:10.1002/joc.1804

Krishnamurti TN, Correa-Torres R, Latif M, Daughenbaugh G (1998) The impact of current and possibly future sea surface temperature anomalies on the frequency of Atlantic hurricanes. Tellus 50:186-210

Pielke RAJ, Landsea CW (1998) Normalized U.S. hurricane damage, 1925-1995. Wea Forecast 13:621-631

Webster PJ, Holland GJ, Curry JA, Chang HR (2005) Changes in tropical cyclone number, duration, and intensity in a warming environment. Science 309(5742):1844-1846. doi:10.1126/ science. 1116448 
William MB (2008) On the changes in the number and intensity of North Atlantic tropical cyclones. J clim 21:1387-1402. doi: 10.1175/2007JCLI1871.1

Wu MC, Yeung KH, Chang WL (2006) Trends in western North Pacific tropical cyclone intensity. Eos Trans AGU 87(48): 537-538. doi:10.1029/2006EO480001
Zhang Q, Liu Q, Wu L (2009) Tropical cyclone damages in China 1983-2006. Bull Am Meteorolog Soc 90:489-495 